\title{
CDISC CDASH Variable Terminology
}

National Cancer Institute

\section{Source}

National Cancer Institute. CDISC CDASH Variable Terminology. NCI Thesaurus. Code C83188.

The terminology subset that includes terms relevant to the Clinical Data Interchange Standards Consortium (CDISC) variables used by the Clinical Data Acquisition Standards Harmonization (CDASH) group. 